\title{
Vorticity generation mechanisms and correct boundary conditions for transverse jet simulation
}

\author{
Y.M. Marzouk, A.F. Ghoniem* \\ Massachusetts Institute of Technology, 77 Massachusetts Ave., Cambridge, MA 02139, USA
}

\begin{abstract}
A three-dimensional vortex element method is developed to simulate the flow field of an incompressible transverse jet at high Reynolds number - in particular, to explore the response of large-scale vortical structures in the jet to actuation. Vorticity produced in the channel wall boundary layer interacts with the jet flow at the nozzle exit, producing both axial and azimuthal perturbations to the primary azimuthal jet vorticity. We derive vorticity boundary conditions that incorporate this interaction, providing a general framework for modeling vorticity associated with actuation. A comparison of centerplane trajectories for unforced transverse jets illustrates the effect of vorticity boundary conditions on the jet near field.
\end{abstract}

Keywords: Vortex dynamics; Vorticity generation; Vortex methods; Jet actuation; Transverse jet

\section{Introduction}

The mixing properties of the transverse jet - a jet issuing normally into a uniform crossflow - are important to a variety of engineering applications. Enhancement of the mixing rate between jet and crossflow can lead to significant improvements in many performance aspects. In gas turbines, for instance, better transverse jet mixing is essential to achieving a wider range of operability, lower emissions, smaller size, and lower noise output. The ultimate objective of this work is to develop control strategies for the transverse jet that manipulate the mixing rate between the jet fluid and the crossflow. To this end, we are developing models that capture the fundamental processes responsible for entrainment and subsequent mixing of fluid from the crossflow into the jet and the response of these processes to actuation.

We construct a computational model of a transverse jet in which actuations may be imposed as time-dependent boundary conditions. We seek to understand the vortex dynamics of the actuated flow, in particular the dynamic response of large-scale vortical structures, such as the counter-rotating vortex pair, to various modes of actuation. An accurate representation of vorticity introduced into the flow field at each timestep is essential for this purpose. Experiments $[4,6]$ and previous computational studies $[11,13]$

*Corresponding author. Tel.: +1 617253 2295; Fax: +1 617 253 5981; E-mail: ghoniem@mit.edu have shown that vorticity from the both the jet boundary layer and the channel wall boundary layer may affect evolution of the transverse jet. This paper derives boundary conditions that account for both sources of vorticity. This construction captures key subtleties in the physics of the unforced transverse jet, and also provides a very general framework with which to describe the vorticity associated with actuation processes.

\section{Numerical formulation}

A three-dimensional vortex element method [3,7-9] is used to develop computational simulations of an unsteady, incompressible transverse jet at large Reynolds number $[10,11]$. These methods solve the equations of motion for inviscid, incompressible flow in vorticity transport form, where $\omega=\nabla \times \mathbf{u}$ :

$$
\begin{aligned}
\frac{D \omega}{D t} & =\boldsymbol{\omega} \cdot \nabla \mathbf{u} \\
\nabla \cdot \mathbf{u} & =0
\end{aligned}
$$

Numerical solution proceeds by discretization of the vorticity field onto overlapping vector elements, each centered at $\chi_{i}^{c}$ with volume $d V_{i}$ and vorticity $\omega_{i}$ :

$$
\boldsymbol{\omega}(\mathbf{x}, t)=\sum_{i}^{N}\left[\omega_{i} d V_{i}\right](t) f_{\delta}\left(\mathbf{x}-\chi_{i}^{c}(t)\right)
$$


The vorticity associated with each element is localized by a radially-symmetric core function $f_{\delta}(\mathbf{r})$ of radius $\delta$, where $f_{\delta}(\mathbf{r}) \equiv \frac{1}{\delta^{3}} f\left(\frac{|\mathbf{r}|}{\delta}\right)$. Here we employ a cubic Gaussian core function proposed by Leonard [9] and shown to yield a second-order discretization [1].

We now discuss boundary conditions particular to the simulation of the transverse jet flow field. In the subsequent expressions, all variables are made dimensionless by $d$, the jet diameter, and $U_{\infty}$, the velocity of the uniform crossflow. The crossflow is directed in the positive $x$ direction; the jet centerline is aligned with the $y$ axis; and the $z$ axis is in the spanwise direction. Except on the disc of the jet orifice, the $x-z$ plane is taken to be a solid wall through which we enforce a no-flow boundary condition. The ratio of the jet velocity to the crossflow velocity is denoted by $r$.

Vorticity produced in the jet boundary layer (i.e., in the pipe below the $y=0$ plane) is represented by a single sheet of azimuthal vorticity. Introducing this vorticity into the flow every $\Delta t_{\text {noz }}$ time units, we divide it among $n$ vortex elements distributed along the edge of the jet nozzle, where $\Delta \theta=2 \pi / n$. These elements have weight

$\left(\omega_{i} d V_{i}\right)_{0}=-\frac{r^{2}}{4} \Delta t_{\mathrm{noz}} \Delta \theta \hat{\mathbf{e}}_{\theta}$

where $\hat{\mathbf{e}}_{\theta}$ is the tangential unit vector in the $x-z$ plane.

Upstream of the jet, vorticity produced in the channel wall boundary layer initially points in the negative spanwise $(-\hat{\mathbf{z}})$ direction. Our interest lies in the interaction of this vorticity with the jet flow immediately around the nozzle edge; in particular, we wish to model channel wall vorticity carried upward by the jet, as this is the vorticity that will affect the evolution of the jet trajectory over the range of $r$. Thus we do not attempt to resolve events in the wall boundary layer away from the jet nozzle, as these have a diminished role in determining jet dynamics for $r>1$ [12].

First, consider the slip of crossflow velocity over the edge of the jet orifice. In polar coordinates $(r, \theta)$ centered at the origin of the $x-z$ plane, the radial component of this slip velocity is canceled locally by an azimuthal vortex sheet of strength $\boldsymbol{\gamma}=-\cos \theta \hat{\mathbf{e}}_{\theta}$. These vortex sheets are shed a distance $r \Delta t_{\text {noz }} / 2$ into the flow every timestep. Again dividing this vorticity over $n=2 \pi / \Delta \theta$ elements distributed along the nozzle edge, we obtain

$\left(\boldsymbol{\omega}_{i} d V_{i}\right)_{1}=-\frac{r}{4} \cos \theta_{i} \Delta t_{\mathrm{noz}} \Delta \theta \hat{\mathbf{e}}_{\theta}$

for the vortex element strengths due to this interaction.

Next we observe that crossflow velocity does not penetrate into the jet at $y=0$. This requires a velocity discontinuity in the $\hat{\mathbf{e}}_{\theta}$ direction, which corresponds to a vortex sheet of strength $\boldsymbol{\gamma}=\sin \theta \hat{\mathbf{e}}_{y}$. Thus the interaction of crossflow vorticity with the jet results in wall-normal vorticity; this idea is confirmed heuristically by considering the tilting of a spanwise material line that encounters either spanwise extremity of the jet. Again, we expect these vortex sheets to be shed at the local flow velocity, i.e., $r \Delta t_{\text {noz }} / 2$ every timestep. Dividing the vorticity over elements along the nozzle edge, we obtain element strengths as:

$\left(\boldsymbol{\omega}_{i} d V_{i}\right)_{2}=\frac{r}{4} \sin \theta_{i} \Delta t_{\mathrm{noz}} \Delta \theta \hat{\mathbf{e}}_{y}$

A final constraint arises from kinematic considerations. In cylindrical coordinates, for vorticity confined to a sheet emanating from the nozzle edge, the solenoidality constraint on the vorticity field $\nabla \cdot \omega=0$ reduces to

$\frac{\partial \omega_{y}}{\partial y}=-2 \frac{\partial \omega_{\theta}}{\partial \theta}$

Each new set of vortex elements represents vorticity in the flow for $0<y<r \Delta t_{\text {noz }} / 2$. We thus introduce elements so that their centers lie at $y=r \Delta t_{\text {noz }} / 4$. With Eq. (6) representing the wall-normal vorticity at $y=0$, enforcing Eq. (7) adds a secondary azimuthal variation to the wallnormal vorticity. Summing Eqs. (4)-(6) and enforcing (7), we obtain the following expression for the total strength of the vortex elements introduced at each timestep:

$$
\begin{aligned}
\boldsymbol{\omega}_{i} d V_{i}= & \left(-\frac{r^{2}}{4}-\frac{r}{4} \cos \theta_{i}\right) \Delta t_{\mathrm{noz}} \Delta \theta \hat{\mathbf{e}}_{\theta} \\
& +\left(\frac{r}{4} \sin \theta_{i}-\frac{r^{2} \Delta t_{\mathrm{noz}}}{8} \sin \theta_{i}\right) \Delta t_{\mathrm{noz}} \Delta \theta \hat{\mathbf{e}}_{y}
\end{aligned}
$$

It is worthwhile to contrast the present vorticity formulation with other vortex models of the transverse jet. Our earlier computational effort [11] neglected vorticity in the crossflow boundary layer, focusing only on the evolution of jet azimuthal vorticity; this approximation is discussed in [11] and its effect will be noted in the results section below. Another recent vortex filament simulation of the unforced transverse jet [2] enforces a no-slip boundary condition along the channel wall by modifying the uniform crossflow with a cubic boundary layer profile near the wall. This boundary layer profile corresponds to a finite vorticity, yet this vorticity is not allowed to evolve, i.e., to obey the dynamics of Eq. (1), nor is it carried into the main flow by the jet.

Jet outflow is represented by a semi-infinite cylindrical vortex sheet of radius $d / 2$ extending from $y=0$ to $y=-\infty$, with strength $\gamma=2 r \hat{\mathbf{e}}_{\theta}$. The vorticity in this cylinder is mollified by a core function identical to that used with the computational vortex elements. This matching is crucial. An unmollified cylindrical vortex sheet, or, equivalently, a uniform distribution of potential sources over the jet orifice with surface source strength $2 r$ [11], yields a singularity in the radial velocity at the nozzle edge when paired with the computational vortex elements.

The crossflow velocity is given by the potential $\phi_{\infty}=x$. Image vortex elements are used to model the behavior of vorticity in the semi-infinite domain, i.e., to enforce noflow through the channel wall $y=0$. Writing the vorticity in the domain componentwise $\boldsymbol{\omega}=\left(\omega_{x}, \omega_{y}, \omega_{z}\right)$, the image vorticity has components $\omega_{\text {img }}=\left(-\omega_{x}, \omega_{y},-\omega_{z}\right)$. 
We now briefly discuss integration of the governing equations using the vortex elements. Vortex elements are described by "sticks," decomposing the element weight $\left[\omega_{i} d V_{i}\right](t)$ into a constant scalar weight $\Gamma_{i}$ times a material length $\delta \chi_{i}(t)$. The vector $\delta \chi_{i}$ points in the direction of the vorticity and is described with two nodes, always with the restriction that the distance between nodes $h=\left|\delta \chi_{i}\right|<0.9 \delta$, where $\delta$ is the core size. Nodes are simply advected by the velocity field:

$$
\frac{d \chi_{i}}{d t}=\mathbf{u}\left(\chi_{i}\right)
$$

Advecting the nodes accounts for deformation of the material line $\delta \chi_{i}$, and thus for stretching and tilting of the vorticity $\omega_{i} d V_{i}$, since vortex lines and material lines coincide.

A second-order predictor/corrector method with timestep control is used for time integration of the ODEs in (9). When $\left|\delta \chi_{i}\right|$ of a given element exceeds $0.9 \delta$, a new node is added at the midpoint of the element, thus splitting the element into two connected elements. Cubic spline interpolants are used to calculate the location of new nodes, and, indeed, to calculate $\delta \chi_{i}$ itself from the tangent vector at each element center. Further detail on the time integration scheme, cubic spline interpolation, and mesh refinement can be found in [11].

\section{Results}

Figs. 1 and 2 show instantaneous velocity vectors and streamlines on the centerplane $z=0$ at $t=3.20$, for sim-

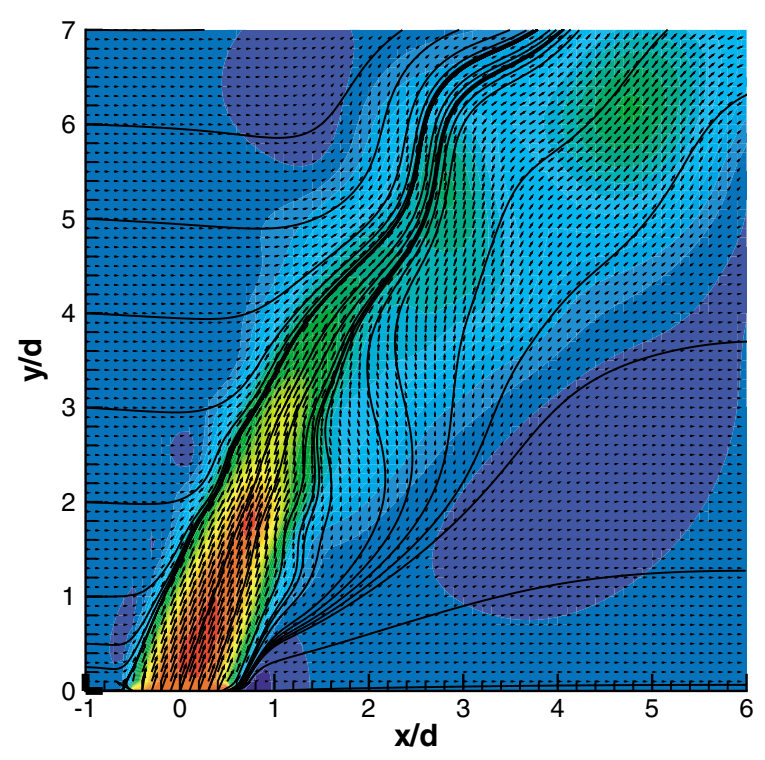

Fig. 1. Velocity streamlines in the centerplane $z=0$, at $\tilde{t}=3.20$, introducing only jet azimuthal vorticity. Contours indicate total velocity magnitude.

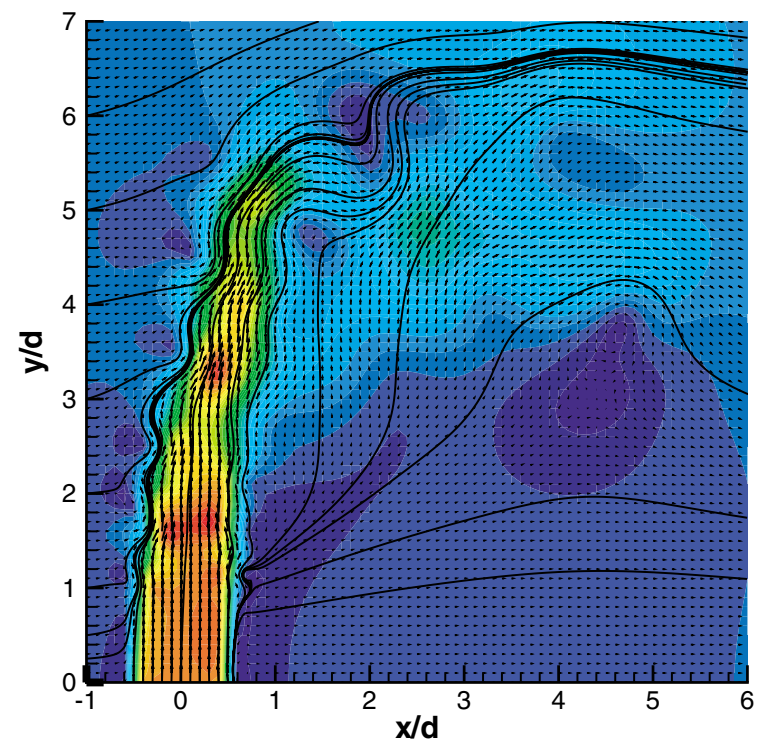

Fig. 2. Velocity streamlines in the centerplane $z=0$, at $\tilde{t}=3.20$, using the vorticity boundary condition in Eq. (8). Contours indicate total velocity magnitude.

ulations with $r=7$. The simulation in Fig. 1 introduces vortex elements containing only jet azimuthal vorticity, i.e., with strengths given by Eq. (4). The simulation in Fig. 2 introduces vortex elements that additionally account for the interaction of channel wall vorticity with the jet, i.e., with strengths given by Eq. (8). Contours indicate the total velocity magnitude $\|\mathbf{u}\|$. In both simulations, the time interval for introducing new filaments at the nozzle was fixed at $\Delta t_{\mathrm{noz}}=0.02$.

The comparison in these two figures clearly illustrates the effect of vorticity boundary conditions on the near field trajectory of the jet. Neglecting vorticity in the jet channel wall boundary layer results in a jet initially angled downstream from the vertical, inconsistent with experimental observation. Modeling the interaction of channel wall vorticity with the jet, however, results in a jet trajectory initially normal to the wall, matching experimental observations and correlations [5].

\section{Conclusions}

Vortex dynamics in the flow field of an incompressible, high-Reynolds number transverse jet are explored with a three-dimensional vortex element method. We construct a simulation capable of mechanistically resolving the vortex dynamics of the actuated flow, providing a clear description of the formation of large-scale vortical structures relevant to mixing [11].

The interaction of vorticity in the crossflow boundary layer with vorticity in the jet boundary layer is modeled and 
used to develop a boundary condition for vorticity entering the flow. The resulting expression contains both azimuthal and wall-normal vorticity, and as a result provides a general and accurate framework with which to describe actuations of the jet flow, e.g., axial pulsation over the range of $r$, swirl, or other helical excitations. A vortex "stick" method is used to compute the evolution of the resulting flow; results show a jet trajectory consistent with analytical models and experimental observations.

Ongoing work uses this model to examine the response of vortical structures over a parameter space of possible actuations.

\section{Acknowledgements}

This work is supported by the US Department of Energy, Office of Science, MICS. Computational support is provided by the National Energy Research Supercomputing Center (NERSC).

\section{References}

[1] Beale JT, Majda A. High-order accurate vortex methods with explicit velocity kernels. J Comput Phys 1985;58:188208.

[2] Cortelezzi L, Karagozian AR. On the formation of the counter-rotating vortex pair in transverse jets. J Fluid Mech
2001;446:347-373.

[3] Cottet G-H, Koumoutsakos PD. Vortex Methods: Theory and Practice. Cambridge University Press, 2000.

[4] Fric TF, Roshko A. Vortical structure in the wake of a transverse jet. J Fluid Mech 1994;279:1-47.

[5] Hasselbrink EF, Mungal MG. Transverse jets and jet flames. Part 1. Scaling laws for strong transverse jets. J Fluid Mech 2001;443:1-25.

[6] Kelso RM, Lim TT, Perry AE. An experimental study of round jets in cross-flow. J Fluid Mech 1996;306:111-144.

[7] Knio OM, Ghoniem AF. Numerical study of a threedimensional vortex method. J Comput Phys 1990;86:75106.

[8] Knio OM, Ghoniem AF. Three-dimensional vortex simulation of rollup and entrainment in a shear layer. J Comput Phys 1991;97:172-223.

[9] Leonard A. Computing three-dimensional incompressible flows with vortex elements. Ann Rev Fluid Mech 1985;17: 523-559.

[10] Marzouk YM, Ghoniem AF. Three-dimensional vortex filament simulation of a transverse jet. Bul Am Phys Soc 2001;46(10)

[11] Marzouk YM, Ghoniem AF. Mechanism of streamwise vorticity formation in a transverse jet. In: 40th Aerospace Sciences Meeting and Exhibit, number AIAA-2002-1063. AIAA, January 2002.

[12] Smith SH, Mungal MG. Mixing, structure, and scaling of the jet in crossflow. J Fluid Mech 1998;357:83-122.

[13] Yuan LL, Street RL, Ferziger JH. Large-eddy simulations of a round jet in crossflow. J Fluid Mech 1999;379:71-104. 